Polymer Journal, Vol. 7, No. 1, pp 79-85 (1975)

\title{
Average Axial Ratio of Stiff Chains
}

\author{
Hiroshi Hoshikawa and Nobuhiko SaItô \\ Department of Applied Physics, Waseda University, Shinjuku-ku \\ Tokyo 160, Japan,
}

Kuniaki NAGAYAMA

Department of Physics, University of Tokyo, Bunkyo-ku

Tokyo 113, Japan.

(Received June 26, 1974)

\begin{abstract}
The shape of stiff chains in dilute solution was considered. An average axial ratio was defined to describe the shape of the stiff chains and calculated using Tagami's model for stiff chains. It was found that the average axial ratio is approximately proportional to $\lambda L$ in the stiff-chain region, where $1 / 2 \lambda$ is the persistence length and $L$ is the contour length of the chain. The limiting behavior agrees with Kuhn's result for a random chain.

KEY WORDS Stiff Chains / Average Shape / Lateral Dimension / Tagami's Model /
\end{abstract}

It is well known that parameters such as the mean-square end-to-end distance $\left\langle R^{2}\right\rangle$ and the mean-square radius of gyration $\left\langle S^{2}\right\rangle$ can characterize the average dimensions of a polymer chain in dilute solution. The shape of a polymer chain, however, is not expressed sufficiently well by $\left\langle R^{2}\right\rangle$ or $\left\langle S^{2}\right\rangle$ alone, since these parameters express the average size rather than the shape. Therefore, we need other parameters to express the shape of polymer chains. $\mathrm{Kuhn}^{1,2}$ considered for Gaussian chains the average loci of various points on the chain contour relative to the end-to-end displacement axis. Hollingsworth, ${ }^{3,4}$ in a study on the same subject, defined the transverse boundary of polymer chains as the average distance of the point which is farthest from the end-to-end displacement axis. Recently, Šolc and Stockmayer, ${ }^{5}$ and Šolc ${ }^{6}$ considered the distribution function $W\left(L_{1}, L_{2}, L_{3}\right)$, where $L_{1}, L_{2}$, and $L_{3}$ are the orthogonal components of the radius of gyration along the principal axes of inertia of the chain. They also examined by Monte Carlo calculations the shape distribution of random chains on simple cubic lattices. The results of these studies suggest that polymer chains in dilute solution are not spherically symmetric in shape.

In this paper, we shall confine ourselves to stiff polymer chains, and consider the average shape of the chains. It is clear from intuition that stiff chains are highly extended in the direction of the end-to-end displacement axis. On the other hand, in the direction transverse to this axis, the distribution of segments is assumed to be cylindrically symmetric. Therefore, there may exist a considerable difference between the two relaxation times of rotational diffusion: the rotation about the axis of the end-to-end displacement and the rotation about the axis perpendicular to that axis. Such a difference, which reflects the average shape of the chain, may be observed experimentally by NMR, nonNewtonian viscosity measurements, and other methods. In addition, since the difference is expected to be larger for the stiff chains than for the random chains, the observation would be easier in the former case.

As mentioned above, stiff chains are extended to the direction of the end vector $\boldsymbol{R}$. Consequently, the appropriate parameter to express the shape of the stiff chain is the average axial ratio $\chi(s)$, which is defined by the following equation:

$$
\chi(s)=\frac{\left\langle H^{2}(s)\right\rangle}{\left\langle R^{2}\right\rangle}
$$




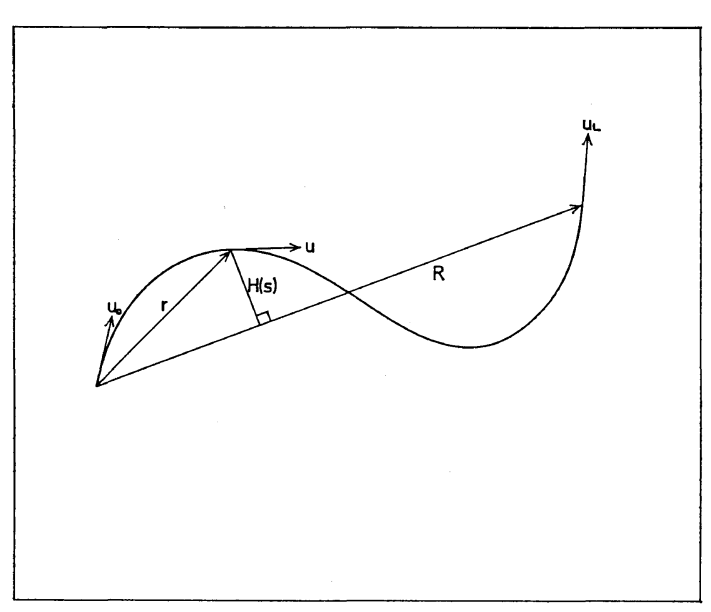

Figure 1. Definitions of $s, \boldsymbol{u}, \boldsymbol{R}, H(s)$, etc.

Here $\left\langle H^{2}(s)\right\rangle$ is the lateral dimension defined by Kuhn: the average square distance of the point of contour length $s$ on the chain from the end-to-end displacement axis. It is clear from Figure 1 that the lateral dimension $\left\langle H^{2}(s)\right\rangle$ is given as follows:

$$
\left\langle H^{2}(s)\right\rangle=\left\langle r^{2}\right\rangle-\left\langle\frac{(\boldsymbol{r} \cdot \boldsymbol{R})^{2}}{R^{2}}\right\rangle
$$

where $\langle\cdots\rangle$ means the average by bivariate distribution function of $\boldsymbol{r}$ and $\boldsymbol{R}$. We may define alternatively another parameter,

$$
\chi^{\prime}(s)=\left\langle\frac{\left\langle H^{2}(s)\right\rangle_{R}}{R^{2}}\right\rangle
$$

where $\langle\cdots\rangle_{\boldsymbol{R}}$ is the average with $\boldsymbol{R}$ kept constant and $\langle\cdots\rangle$ is the average over $\boldsymbol{R}$. Mathematically the quantity (1) can be calculated much more easily than eq 3 . Thus we shall discuss exclusively the quantity defined by eq 1 .

\section{AVERAGE AXIAL RATIO OF STIFF CHAINS}

From the various models of stiff chains, ${ }^{7-10}$ we choose to use Tagami's model, ${ }^{10}$ since its distribution function of end-to-end distances is given by a closed form. In this model, the Brownian motion of stiff chains is compared to the $\mathrm{O}-\mathrm{U}$ process (Ornstein-Uhlenbeck process) of one Brownian particle. The Wiener process of one Brownian particle is equivalent to a random chain. On the other hand, in the $\mathrm{O}-$ $\mathrm{U}$ process, due to the diffusion in the momentum space, the direction of motion of the particle is no longer random, which means in polymer language that the chain becomes stiff or semiflexible. The correspondence is as follows: Let the time $s$ in the $\mathrm{O}-\mathrm{U}$ process correspond to the contour length $s$ of the chain; then the path of the particle corresponds to the configuration of the chain, and the momentum of the particle at time $s$ corresponds to the derivative of the position vector of the point $s$ on the chain contour. An interpretation of this model in terms of the elasticity of the chain will be given in the appendix.

The distribution function of the $\mathrm{O}-\mathrm{U}$ process of one Brownian particle obeys the well known Fokker-Planck equation

$$
\begin{aligned}
& \frac{\partial}{\partial s} f\left(\boldsymbol{r}, \boldsymbol{u}, s \mid \boldsymbol{r}_{0}, \boldsymbol{u}_{0}\right)=\operatorname{div}_{u}\left(q \cdot \operatorname{grad}_{u} f\right) \\
& \quad+\operatorname{div}_{u}(\beta f \boldsymbol{u})-\boldsymbol{u} \cdot \operatorname{grad}_{r} f
\end{aligned}
$$

where

$$
\begin{aligned}
& \langle\Delta \boldsymbol{u}\rangle=-\beta \boldsymbol{u} \Delta s \\
& \left\langle\Delta u^{2}\right\rangle=6 q \Delta s
\end{aligned}
$$

In order to be able to regard eq 4 as the equation of the distribution function of stiff chains, we first notice that the elongation and the contraction of stiff chains are assumed to be small. Therefore the requirement

$$
\langle\boldsymbol{u}(s) \cdot \boldsymbol{u}(s)\rangle=1
$$

is natural. From eq 5 , we have

$$
\frac{1}{6 q}=\frac{\Delta s}{\left\langle\Delta u^{2}\right\rangle} \sim \frac{\Delta s}{\left\langle\theta^{2}\right\rangle}=\frac{1}{2} \frac{1}{2 \lambda}
$$

where $\theta$ is the angle between $\boldsymbol{u}(s)$ and $\boldsymbol{u}(s+\Delta s)$, and $1 / 2 \lambda$ is the so-called persistence length. From eq 6 , we see that $q / \beta=1 / 3$; consequently $\beta=2 \lambda$. (Notice that in the theory of Brownian motion of one particle, we have $q=\beta k T / m$, according to the equipartition law of energy). Since it is conventional to use $\lambda$ in the theory of stiff chains, we use $\lambda$ instead of $q$ and $\beta$.

The elementary solution of eq 4 is given by Chandrasekhar ${ }^{11}$ as follows: 
Average Axial Ratio of Stiff Chains

$$
\begin{aligned}
& f\left(\boldsymbol{r}, \boldsymbol{u}, s \mid \boldsymbol{r}_{0}, \boldsymbol{u}_{0}\right) \\
& \quad=\frac{e^{6 \lambda s}}{8 \pi^{3} \Delta^{3 / 2}} \exp \left[-\frac{1}{2 \Delta}\left\{a\left(e^{2 \lambda s} \boldsymbol{u}-\boldsymbol{u}_{0}\right)^{2}\right.\right. \\
& \quad+2 h\left(e^{2 \lambda s} \boldsymbol{u}-\boldsymbol{u}_{0}\right)\left(\boldsymbol{r}+\frac{\boldsymbol{u}}{2 \lambda}-\boldsymbol{r}_{0}-\frac{\boldsymbol{u}_{0}}{2 \lambda}\right) \\
& \left.\left.\quad+b\left(\boldsymbol{r}+\frac{\boldsymbol{u}}{2 \lambda}-\boldsymbol{r}_{0}-\frac{\boldsymbol{u}_{0}}{2 \lambda}\right)^{2}\right\}\right]
\end{aligned}
$$

where

$$
\begin{aligned}
& a=\frac{s}{3 \lambda}, \quad b=\frac{1}{3}\left(e^{4 \lambda s}-1\right), \\
& h=-\frac{1}{3 \lambda}\left(e^{2 \lambda s}-1\right), \quad \Delta=a b-h^{2}
\end{aligned}
$$

If necessary, we shall write $a(s), b(s), h(s)$, and $\Delta(s)$ respectively to indicate the $s$ dependence.

First we have to obtain the bivariate distribution function $F(\boldsymbol{r}, s ; \boldsymbol{R}, L)$. This is written as follows, by making use of the elementary solution $f\left(\boldsymbol{r}, \boldsymbol{u}, s \mid \boldsymbol{r}_{0}, \boldsymbol{u}_{0}\right)$ given above (see Figure 1):

$$
\begin{gathered}
F(\boldsymbol{r}, s ; \boldsymbol{R}, L)=\iiint f\left(\boldsymbol{R}, \boldsymbol{u}_{L}, L-s \mid \boldsymbol{r}, \boldsymbol{u}\right) \\
\quad \times f\left(\boldsymbol{r}, \boldsymbol{u}, s \mid \boldsymbol{o}, \boldsymbol{u}_{0}\right) g\left(\boldsymbol{u}_{0}\right) d \boldsymbol{u}_{L} d \boldsymbol{u} d \boldsymbol{u}_{0} \\
\quad=\int \boldsymbol{G}\left(\boldsymbol{r}, s ; \boldsymbol{R}, L \mid \boldsymbol{u}_{0}\right) g\left(\boldsymbol{u}_{0}\right) d \boldsymbol{u}_{0}
\end{gathered}
$$

where $G\left(\boldsymbol{r}, s ; \boldsymbol{R}, L \mid \boldsymbol{u}_{0}\right)$ is the conditional bivariate distribution function of $\boldsymbol{r}$ and $\boldsymbol{R}$ with $\boldsymbol{u}_{0}$ fixed, and $g\left(\boldsymbol{u}_{0}\right)$ is the distribution function of $\boldsymbol{u}_{0}$, which is assumed to be of the form

$$
g\left(\boldsymbol{u}_{0}\right)=\frac{1}{4 \pi} \delta\left(\left|\boldsymbol{u}_{0}\right|-1\right)
$$

The function $\boldsymbol{G}\left(\boldsymbol{r}, s ; \boldsymbol{R}, L \mid \boldsymbol{u}_{0}\right)$ is calculated from eq 8 and 10 to obtain

$$
\begin{aligned}
G(\boldsymbol{r}, & \left.s ; \boldsymbol{R}, L \mid \boldsymbol{u}_{0}\right)=\left(\frac{1}{8 \pi^{3}}\right)^{2}\left\{\frac{\pi^{2}}{\mu \nu \Delta(s) \Delta(L-S)}\right\}^{3 / 2} \\
& \times \exp \left[-\omega r^{2}+\boldsymbol{D} \cdot \boldsymbol{r}+\tau\right]
\end{aligned}
$$

where $\mu, \nu, \omega, D$, and $\tau$ are defined by

$$
\begin{aligned}
& \mu=\frac{\alpha_{1}(s)}{2 \Delta(s)}+\frac{\alpha_{2}(L-s)}{2 \Delta(L-s)} \\
& \nu=\frac{\alpha_{1}(L-s)}{2 \Delta(L-s)}-\frac{\alpha_{3}^{2}(L-s)}{4 \mu \Delta^{2}(L-s)} \\
& \omega=-\frac{1}{4 \nu}\left\{\frac{\alpha_{3}(L-s) \alpha_{4}(s)}{2 \mu \Delta(s) \Delta(L-s)}\right\}^{2}-\frac{1}{4 \nu}\left\{\frac{\alpha_{3}(L-s) \alpha_{5}(L-s)}{2 \mu \Delta^{2}(L-s)}-\frac{\alpha_{4}(L-s)}{\Delta(L-s)}\right\}^{2} \\
& -\frac{\alpha_{3}(L-s) \alpha_{4}(s)}{4 \mu \nu \Delta(s) \Delta(L-s)}\left\{\frac{\alpha_{3}(L-s) \alpha_{5}(L-s)}{2 \mu \Delta^{2}(L-s)}-\frac{\alpha_{4}(L-s)}{\Delta(L-s)}\right\}-\frac{\alpha_{4}{ }^{2}(s)}{4 \mu \Delta^{2}(s)} \\
& -\frac{\alpha_{4}(s) \alpha_{5}(L-s)}{2 \mu \Delta(s) \Delta(L-s)}-\frac{\alpha_{5}{ }^{2}(L-s)}{4 \mu \Delta^{2}(L-s)}+\frac{b(s)}{2 \Delta(s)}+\frac{b(L-s)}{2 \Delta(L-s)} \\
& \boldsymbol{D}=\left[-\frac{\alpha_{3}(s) \alpha_{4}(s)}{2 \nu}\left\{\frac{\alpha_{3}(L-s)}{2 \mu \Delta(s) \Delta(L-s)}\right\}^{2}-\frac{\alpha_{3}(s) \alpha_{4}(s)}{2 \mu \Delta^{2}(s)}-\frac{\alpha_{3}(s) \alpha_{5}(L-s)}{2 \mu \Delta(s) \Delta(L-s)}\right. \\
& \left.-\frac{\alpha_{3}(s) \alpha_{3}(L-s)}{4 \mu \nu \Delta(s) \Delta(L-s)}\left\{\frac{\alpha_{3}(L-s) \alpha_{5}(L-s)}{2 \mu \Delta^{2}(L-s)}-\frac{\alpha_{4}(L-s)}{\Delta(L-s)}\right\}+\frac{\alpha_{5}(s)}{\Delta(s)}\right] \boldsymbol{u}_{0} \\
& -\left[\frac{1}{2 \nu}\left\{\frac{\alpha_{3}(L-s) \alpha_{5}(L-s)}{2 \mu \Delta^{2}(L-s)}-\frac{\alpha_{4}(L-s)}{\Delta(L-s)}\right\}^{2}+\frac{\alpha_{4}(s) \alpha_{5}(L-s)}{2 \mu \Delta(s) \Delta(L-s)}+\frac{\alpha_{5}^{2}(L-s)}{2 \mu \Delta^{2}(L-s)}\right. \\
& \left.+\frac{\alpha_{3}(L-s) \alpha_{4}(s)}{4 \mu \nu \Delta(s) \Delta(L-s)}\left\{\frac{\alpha_{3}(L-s) \alpha_{5}(L-s)}{2 \mu \Delta^{2}(L-s)}-\frac{\alpha_{4}(L-s)}{\Delta(L-s)}\right\}-\frac{b(L-s)}{\Delta(L-s)}\right] \boldsymbol{R} \\
& \tau=-\left[-\frac{1}{4 \nu}\left\{\frac{\alpha_{3}(s) \alpha_{3}(L-s)}{2 \mu \Delta(s) \Delta(L-s)}\right\}^{2}-\frac{\alpha_{3}{ }^{2}(s)}{4 \mu \Delta^{2}(s)}+\frac{\alpha_{2}(s)}{2 \Delta(s)}\right] u_{0}^{2} \\
& +\left[\frac{1}{4 \nu}\left\{\frac{\alpha_{3}(L-s) \alpha_{5}(L-s)}{2 \mu \Delta^{2}(L-s)}-\frac{\alpha_{4}(L-s)}{\Delta(L-s)}\right\}^{2}+\frac{\alpha_{5}{ }^{2}(L-s)}{4 \mu \Delta^{2}(L-s)}-\frac{b(L-s)}{2 \Delta(L-s)}\right] R^{2} \\
& +\left[\frac{\alpha_{3}(s) \alpha_{3}(L-s)}{4 \mu \nu \Delta(s) \Delta(L-s)}\left\{\frac{\alpha_{3}(L-s) \alpha_{5}(L-s)}{2 \mu \Delta^{2}(L-s)}-\frac{\alpha_{4}(L-s)}{\Delta(L-s)}\right\}+\frac{\alpha_{3}(s) \alpha_{5}(L-s)}{2 \mu \Delta(s) \Delta(L-s)}\right] \boldsymbol{u}_{0} \cdot \boldsymbol{R}+6 \lambda L
\end{aligned}
$$

Furthermore, 
H. Hoshikawa, N. Saitô, and K. Nagayama

$$
\begin{aligned}
& \alpha_{1}(s)=a(s) e^{4 \lambda s}+\frac{h(s) e^{2 \lambda s}}{\lambda}+\frac{b(s)}{4 \lambda^{2}} \\
& \alpha_{2}(s)=a(s)+\frac{h(s)}{\lambda}+\frac{b(s)}{4 \lambda^{2}} \\
& \alpha_{3}(s)=a(s) e^{2 \lambda s}+\frac{h(s) e^{2 \lambda s}}{2 \lambda}+\frac{h(s)}{2 \lambda}+\frac{b(s)}{4 \lambda^{2}} \\
& \alpha_{4}(s)=h(s) e^{2 \lambda s}+\frac{b(s)}{2 \lambda} \\
& a_{5}(s)=h(s)+\frac{b(s)}{2 \lambda}
\end{aligned}
$$

The conditional distribution function of $\boldsymbol{r}, F_{0}(\boldsymbol{r}, s \mid \boldsymbol{R})$, with $\boldsymbol{R}$ fixed, is calculated by making use of the relation

$$
F_{0}(\boldsymbol{r}, s \mid \boldsymbol{R})=\frac{F(\boldsymbol{r}, s ; \boldsymbol{R}, L)}{f_{0}(\boldsymbol{R})}
$$

where $f_{0}(\boldsymbol{R})$ is the distribution function of $\boldsymbol{R}$ :

$$
f_{0}(\boldsymbol{R})=\iint f\left(\boldsymbol{R}, \boldsymbol{u}_{L}, L \mid \boldsymbol{o}, \boldsymbol{u}_{0}\right) g\left(\boldsymbol{u}_{0}\right) \mathrm{d} \boldsymbol{u}_{L} \mathrm{~d} \boldsymbol{u}_{0} .
$$

The explicit expression of eq 16 is given from eq 8 and 11 ,

$$
\begin{aligned}
f_{0}(\boldsymbol{R})= & \left\{\frac{6 \lambda^{2}}{\pi\left(4 \lambda L-3+4 e^{-2 \lambda L}-e^{-4 \lambda L}\right)}\right\}^{3 / 2} \exp \left[-\frac{3\left\{4 \lambda^{2} R^{2}+\left(1-e^{-2 \lambda L}\right)^{2}\right\}}{2\left(4 \lambda L-3+4 e^{-2 \lambda L}-e^{-4 \lambda L}\right)}\right] \\
& \times \frac{4 \lambda L-3+4 e^{-2 \lambda L}-e^{-4 \lambda L}}{6 \lambda\left(1-e^{-2 \lambda L}\right) R} \sinh \frac{6 \lambda\left(1-e^{-2 \lambda L}\right) R}{4 \lambda L-3+4 e^{-2 \lambda L}-e^{-4 \lambda L}}
\end{aligned}
$$

From eq 15-17, the lateral dimension with $\boldsymbol{R}$ fixed $\left\langle H^{2}(s)\right\rangle_{R}$, i.e., the mean-square of $H(s)$ with $\boldsymbol{R}$ fixed, can be calculated to be

$$
\begin{aligned}
\left\langle H^{2}(s\rangle_{R}=\right. & \int H^{2}(s) F_{0}(\boldsymbol{r}, s \mid \boldsymbol{R}) \mathrm{d} \boldsymbol{r}=\frac{1}{f_{0}(\boldsymbol{R})}\left(\frac{1}{8 \pi^{3}}\right)^{2}\left\{\frac{\pi^{3}}{\mu \nu \omega \Delta(s) \Delta(L-s)}\right\}^{3 / 2} \frac{1}{R\left(2 \omega \eta_{3}-\xi_{1} \xi_{2}\right)} \\
& \times \exp \left[\frac{\xi_{1}{ }^{2}}{4 \omega}-\eta_{1}+\left(\frac{\xi_{2}{ }^{2}}{4 \omega}+\eta_{2}\right) R^{2}+6 \lambda L\right]\left[2 \sinh \frac{R\left(2 \omega \eta_{3}-\xi_{1} \xi_{2}\right)}{2 \omega}+\frac{2 \xi_{1}{ }^{2}}{R\left(2 \omega \eta_{3}-\xi_{1} \xi_{2}\right)}\right. \\
& \left.\times\left\{\cosh \frac{R\left(2 \omega \eta_{3}-\xi_{1} \xi_{2}\right)}{2 \omega}-\frac{2 \omega}{R\left(2 \omega \eta_{3}-\xi_{1} \xi_{2}\right)} \sinh \frac{R\left(2 \omega \eta_{3}-\xi_{1} \xi_{2}\right)}{2 \omega}\right\}\right]
\end{aligned}
$$

where $\xi_{1}, \xi_{2}, \eta_{1}, \eta_{2}$, and $\eta_{3}$ are defined by

$$
\begin{aligned}
& D=\xi_{1} u_{0}-\xi_{2} R \\
& \tau=-\eta_{1} u_{0}{ }^{2}+\eta_{2} R^{2}+\eta_{3} u_{0} \cdot R+6 \lambda L
\end{aligned}
$$

On the other hand, the lateral dimension $\left\langle H^{2}(s)\right\rangle$ is obtained by averaging $H^{2}(s)$ over $\boldsymbol{r}$ and $\boldsymbol{R}$ making use of eq 10 ; thus

$$
\begin{aligned}
\left\langle H^{2}(s)\right\rangle & =\iint H^{2}(s) F(\boldsymbol{r}, s ; \boldsymbol{R}, L) \mathrm{d} \boldsymbol{r} \mathrm{d} \boldsymbol{R} \\
& =\left(\frac{1}{8 \pi^{3}}\right)\left\{\frac{\pi^{3}}{\mu \nu \omega \Delta(s) \Delta(L-s)}\right\}^{3 / 2} \frac{1}{2 \omega \eta_{3}-\xi_{1} \xi_{2}} \exp \left[\frac{\xi_{1}{ }^{2}}{4 \omega}-\eta_{1}+6 \lambda L\right]\left[\left(\frac{-16 \omega \pi^{3}}{4 \omega \eta_{2}+\xi_{2}{ }^{2}}\right)^{1 / 2}\right. \\
& \times \exp \left[-\frac{\left(2 \omega \eta_{3}-\xi_{1} \xi_{2}\right)^{2}}{4 \omega\left(4 \omega \eta_{2}+\xi_{2}{ }^{2}\right)}\right]\left\{-\frac{4 \omega \eta_{3}-2 \xi_{1} \xi_{2}}{4 \omega \eta_{2}+\xi_{2}{ }^{2}}+\frac{2 \xi_{1}{ }^{2}}{2 \omega \eta_{3}-\xi_{1} \xi_{2}}\right\}
\end{aligned}
$$




$$
\left.-\frac{16 \pi^{3 / 2} \omega \xi_{1}{ }^{2}}{\left(2 \omega \eta_{3}-\xi_{1} \xi_{2}\right)^{2}} \Phi\left(\frac{2 \omega \eta_{3}-\xi_{1} \xi_{2}}{\left\{-4 \omega\left(4 \omega \eta_{2}+\xi_{2}^{2}\right)\right\}^{1 / 2}}\right)\right]
$$

where

$$
\Phi(x)=\int_{0}^{x} e^{t^{2}} d t
$$

In deriving eq 20 , we have made use of the approximation

$$
\int_{0}^{\frac{2 \omega \eta_{3}-\xi_{1} \xi_{2}}{\left\{-4 \omega\left(4 \omega \eta_{2}+\xi_{2}^{2}\right)\right\}^{1 / 2}}} e^{-t^{2}} d t=0
$$

which is correct in both rod and random coil limit, since

$$
\frac{2 \omega \eta_{3}-\xi_{1} \xi_{2}}{\left\{-4 \omega\left(4 \omega \eta_{2}+\xi_{2}^{2}\right)\right\}^{1 / 2}} \longrightarrow 0
$$

holds in both limits. The average axial ratio $\chi(s)$ is readily obtained from eq 1 and 20 :

$$
\chi(s)=\frac{\left\langle H^{2}(s)\right\rangle}{\left\langle R^{2}\right\rangle}
$$

where $\left\langle R^{2}\right\rangle$ is the well known mean-square endto-end distance, and is given by

$$
\left\langle R^{2}\right\rangle=\frac{2 \lambda L-1+e^{-2 \lambda L}}{2 \lambda^{2}}
$$

The quantities $\left\langle H^{2}(s)\right\rangle_{R},\left\langle H^{2}(s)\right\rangle$, and $\chi(s)$ thus obtained in eq 18,20 , and 23 are general expressions which hold at any point on the chain contour. In particular, the values of these parameters at the mid-point on the chain contour are practically useful. In Figures 2 and 3, $\left\langle H^{2}(L / 2)\right\rangle$ and $\left\langle H^{2}(L / 2)\right\rangle_{R}$, and $\chi(L / 2)$ are shown as function of $\lambda L$.

The limiting behavior of these parameters in the case of $\lambda L \rightarrow \infty$, i.e., in the random coil limit, is

$$
\begin{aligned}
& \lim _{\lambda L \rightarrow \infty}\left\langle H^{2}\left(\frac{L}{2}\right)\right\rangle_{R}=\frac{1}{6} \frac{L}{\lambda} \\
& \lim _{\lambda L \rightarrow \infty}\left\langle H^{2}\left(\frac{L}{2}\right)\right\rangle=\frac{1}{6} \frac{L}{\lambda} \\
& \lim _{\lambda L \rightarrow \infty} \chi\left(\frac{L}{2}\right)=\frac{1}{6}
\end{aligned}
$$

These results agree with Kuhn's result. On the other hand, in the limit $\lambda L \rightarrow 0$, i.e., in the rigid rod limit, all of these parameters approach zero.

Figure 3. $\log \chi^{1 / 2}(L / 2) v s . \log \lambda L$. The broken line corresponds to $\lim _{\lambda L \rightarrow \infty} \chi^{1 / 2}(L / 2)=\log (1 / 6)^{1 / 2}$.

\section{DISCUSSION}

As shown in Figure 2, $\left\langle H^{2}(L / 2)\right\rangle^{1 / 2} / L$ has a maximum in the region $\lambda L \sim 1$, or in the stiff chain region. This is understood as follows: in the region $\lambda L \ll 1$, i.e., in the rigid rod region, we have evidently $\left\langle H^{2}(L / 2)\right\rangle^{1 / 2} / L \ll 1$; as $\lambda L$ increases, the bending of the chain becomes larger and therefore $\left\langle H^{2}(L / 2)\right\rangle^{1 / 2} / L$ increases; in the region $\lambda L \gg 1$, i.e., in the random coil region, 
not only the mid-point but any point on the chain contour can be far from the end-to-end displacement axis, thus we expect that $\left\langle H^{2}(L /\right.$ 2) $\rangle^{1 / 2} / L$ decreases and tends to $(1 / 6 \lambda L)^{1 / 2}$ in the limit $\lambda L \rightarrow \infty$, as shown in eq $25 \mathrm{~b}$.

In Figure 2 the quantity $\left\langle H^{2}(L / 2)\right\rangle_{R}^{1 / 2} / L$ is also shown. We can see that in the region $\lambda L \ll 1$, $\left\langle H^{2}(L / 2)\right\rangle_{R=L}^{1 / 2} / L$ coincides with $\left\langle H^{2}(L / 2)\right\rangle^{1 / 2} / L$, because in this region the contribution of the highly extended state to the quantity $\left\langle H^{2}(L / 2)\right\rangle$ is large. As $\lambda L$ increases, the difference between $\left\langle H^{2}(L / 2)\right\rangle$ and $\left\langle H^{2}(L / 2)\right\rangle_{R=L}$ becomes larger, but when $\lambda L$ increases further $(\lambda L \gg 1)$, the difference becomes smaller and the three functions, $\left\langle H^{2}(L /\right.$ $2)\rangle_{R=L},\left\langle H^{2}(L / 2)\right\rangle_{R \ll L}$, and $\left\langle H^{2}(L / 2)\right\rangle$ coincide with one another, in accordance with eq $25 \mathrm{a}$ and $25 b$.

We see in Figure 3 that the function $\chi(L / 2)$ increases with increasing $\lambda L$, and tends to $1 / 6$ for the random coil limit, which implies that $\chi(s)$ is an appropriate parameter to describe the shape of stiff chains. It should be noted that in the stiff chain region $\left(10^{-1} \leq \lambda L \leqq 1\right), \chi(L / 2)$ is approximately proportional to $\lambda L$.

Acknowledgment. The authors wish to thank Professor Akiyoshi Wada for suggesting this problem.

\section{APPENDIX}

The distribution function of the $\mathrm{O}-\mathrm{U}$ process of one Brownian particle can be written in the form of a path integra $1^{12}$ as

$$
f\left(\boldsymbol{u}_{2}, s_{2} \mid \boldsymbol{u}_{1}, s_{1}\right)=\int \exp \left[-\frac{1}{2 \zeta k T} \int_{s_{1}}^{s_{2}} L \mathrm{ds}\right] D \boldsymbol{u}
$$

where $\zeta$ is the friction constant. The Lagrangian $L$ is given by

$$
L=\frac{1}{2}(m \dot{u}+\zeta u)^{2}
$$

The distribution function of Tagami's model can also be written in the path integral form:

$$
f\left(\boldsymbol{u}_{2}, s_{2} \mid \boldsymbol{u}_{1}, s_{1}\right)=\int \exp \left[-\frac{1}{k T} \int_{s_{1}}^{s_{2}} L \mathrm{ds}\right] D \boldsymbol{u}
$$

where the Lagrangian $L$ is given by

$$
\begin{aligned}
L & =\frac{1}{2}\left\{\left(\frac{3 k T}{4 \lambda}\right)^{1 / 2} \dot{u}+(3 \lambda k T)^{1 / 2} \boldsymbol{u}\right\}^{2} \\
& =\frac{3 k T}{8 \lambda} \dot{\boldsymbol{u}}^{2}+\frac{3 k T}{4}(\dot{\boldsymbol{u}} \cdot \boldsymbol{u}+\boldsymbol{u} \cdot \dot{\boldsymbol{u}})+\frac{3 \lambda k T}{2} \boldsymbol{u}^{2}
\end{aligned}
$$

We can easily obtain from eq A-3 and A-4 the Fokker-Planck equation of Tagami's model by the method described in ref 12 . Comparing eq A-3 with the path integral representation for Kratky-Porod model of stiff chains, ${ }^{9}$ we see that the term of $\dot{u}^{2}$ in the Lagrangian is the bending elastic energy, but its coefficient is different from that of the Kratky-Porod model by the factor $3 / 2$. The remaining terms are regarded as the potential which characterizes Tagami's model. The contribution of the second term of the right hand side of eq A-4 to the path integral is

$$
\int_{s_{1}}^{s_{2}}(\dot{\boldsymbol{u}} \cdot \boldsymbol{u}+\boldsymbol{u} \cdot \dot{\boldsymbol{u}}) \mathrm{d} s=u^{2}\left(s_{2}\right)-u^{2}\left(s_{1}\right)
$$

This term can be interpreted as the coupling energy between bending and stretching. The third term of the right hand side of eq A-4 is regarded as the stretching elastic energy of Tagami's model. This term can be rewritten as

$$
u^{2}=(|\boldsymbol{u}|-1)^{2}+2|\boldsymbol{u}|-1
$$

where the first term gives rise to the stretching elastic energy in Hookian approximation, and the remaining terms are some sort of nonHookian terms. Contrary to the usual mechanical model, ${ }^{9}$ however, the stretching elastic coefficient is temperature-dependent as in the rubber elasticity provided that $\lambda$ is temperatureindependent. It seems at first sight that the chain can be elongated to infinity in the limit $\lambda L \rightarrow 0$, and the elongation of the chain is zero in the limit $\lambda L \rightarrow \infty$, since the coefficient of $u^{2}$ is proportional to $\lambda$. However, it can be easily shown that

$$
\left\langle u^{2}\right\rangle=1
$$

and

$$
\left\langle u^{4}\right\rangle=\left\{\begin{array}{lll}
\frac{5}{3} & \text { for } & \lambda L \rightarrow \infty \\
1 & \text { for } & \lambda L \rightarrow 0
\end{array}\right.
$$

In other words the fluctuation of the chain elongation is Gaussian in the random coil limit 
$(\lambda L \rightarrow \infty)$, but is absent in the rigid rod limit $(\lambda L \rightarrow 0)$. The latter fact can be understood from the form of the Lagrangian (A-4). In the limit $\lambda \rightarrow 0$, the coefficient $3 \lambda k T / 2$ of the last term (stretching elastic constant) is zero, and therefore the chain seems to be stretched easily, but by virtue of the large coefficient $3 k T / 8 \lambda$ of the first term, $\dot{\boldsymbol{u}}$ should be nearly zero $(\dot{\boldsymbol{u}} \simeq 0)$. In other words, since $\left\langle\dot{u}^{2}\right\rangle=1$, the chain is rodlike.

\section{REFERENCES}

1. W. Kuhn, Kolloid. Z., 68, 2 (1934).

2. W. Kuhn, J. Polym. Sci., 1, 380 (1946).
3. C. A. Hollingsworth, J. Chem. Phys., 16, 544 (1948).

4. C. A. Hollingsworth, ibid., 17, 97 (1949).

5. K. Šolc and W. H. Stockmayer, ibid., 54, 2759 (1971).

6. K. Šolc, ibid., 55, 335 (1971).

7. O. Kratky and G. Porod, Rec. Trav. Chim., 68, 1106 (1949).

8) R. A. Harris and J. E. Hearst, J. Chem. Phys., 44, 2595 (1966).

9. N. Saitô, K. Takahashi, and Y. Yunoki, $J$. Phys. Soc. Japan, 22, 219 (1967).

10. Y. Tagami, Macromolecules, 2,8 (1969).

11. S. Chandrasekhar, Rev. Mod. Phys., 15, 1 (1943).

12. N. Saitô and M. Namiki, Prog. Theor. Phys., 16, 71 (1956). 